\title{
Condição oral de dependentes químicos em um município do estado da Paraíba
}

\author{
Oral condition of chemical dependents in a municipality in the state of Paraíba
}

Condición oral de los dependientes químicos en un municipio del estado de Paraíba

Bruna Mayara Gonçalves de Barros ${ }^{1 *}$, Micaella Fernandes Farias ${ }^{1}$, José Jhenikártery Maia de Oliveira ${ }^{1}$, Laís Guimarães Pinto ${ }^{1}$, Giullianna de Andrade Silva ${ }^{1}$, Kalygia Gabrielle Cavalcanti Alves de Souza1 ${ }^{1}$, Laudenice de Lucena Pereira1 ${ }^{1}$, Maria Cristina Tavares de Medeiros Honorato'.

\section{RESUMO}

Objetivo: Identificar a condição oral dos dependentes químicos atendidos em um Centro de Especialidades Odontológicas (CEO), traçar o perfil sociodemográfico, determinando a prevalência de lesões/alterações estomatológicas nestes pacientes. Métodos: Trata-se de um trabalho descritivo com abordagem quantitativa e bibliográfica, realizado no município de João Pessoa-PB. A amostra constituiu-se de 90 prontuários. Os dados foram coletados em ficha clínica elaborada para este fim, tabulados e submetidos à análise descritiva. Resultados: Há uma maior frequência de tabagistas (41\%), seguida de etilistas/ tabagistas (39\%), apenas etilistas (18\%) e usuário de drogas ilícitas (1\%). A maioria (60\%) destes fazia uso de drogas por mais de 10 anos, numa periodicidade de mais de três vezes por semana. Houve maior prevalência de lesões de menor impacto à saúde (41\%), seguida de pacientes sem alterações estomatológicas (32\%) e lesões de maior impacto à saúde (27\%). Conclusão: Apesar de ser considerada população de risco para o desenvolvimento do câncer de boca, o carcinoma de células escamosas (CCE) foi menos prevalente que a ocorrência de lesões com potencial de malignidade, predominando as alterações estomatológicas de menor impacto à saúde geral dos pacientes.

Palavras-chave: Dependentes químicos, Saúde bucal, Medicina bucal.

\begin{abstract}
Objective: To identify the oral condition of drug addicts treated at a Dental Specialties Center (CEO), trace the sociodemographic profile, determining the prevalence of lesions / stomatological changes in these patients. Methods: This is a descriptive work with a quantitative and bibliographic approach, carried out in the city of João Pessoa-PB. The sample consisted of 90 medical records. The data were collected in a clinical form prepared for this purpose, tabulated and submitted to descriptive analysis. Results: There is a higher frequency of smokers $(41 \%)$, followed by alcoholics / smokers (39\%), only alcoholics (18\%) and users of illicit drugs (1\%). The majority $(60 \%)$ of them used drugs for more than 10 years, more than three times a week. There was a higher prevalence of injuries with less impact on health $(41 \%)$, followed by patients without stomatological changes (32\%) and injuries with greater impact on health (27\%). Conclusion: Despite being considered a population at risk for the development of oral cancer, squamous cell carcinoma (SCC) was less prevalent than the occurrence of lesions with malignant potential, with predominance of stomatological changes with less impact on the general health of patients.
\end{abstract}

Keywords: Drug users, Oral health, Oral medicine.

${ }^{1}$ Centro Universitário de João Pessoa (UNIPÊ), João Pessoa - PB.

*E-mail: micaellaff@gmail.com

SUBMETIDO EM: 4/2020

ACEITO EM: 5/2020

PUBLICADO EM: 7/2020

REAS/EJCH | Vol.Sup.n.52 | e3695 | DOI: https://doi.org/10.25248/reas.e3695.2020 Página 1 de 10 


\section{RESUMEN}

Objetivo: Identificar la condición oral de los drogadictos tratados en un Centro de Especialidades Dentales (CEO), rastrear el perfil sociodemográfico, determinando la prevalencia de lesiones / cambios estomatológicos en estos pacientes. Métodos: Se trata de un trabajo descriptivo con enfoque cuantitativo y bibliográfico, realizado en la ciudad de João Pessoa-PB. La muestra consistió en 90 registros médicos. Los datos fueron recolectados en una forma clínica preparada para este propósito, tabulada y sometida a análisis descriptivo. Resultados: Hay una mayor frecuencia de fumadores (41\%), seguidos de alcohólicos / fumadores (39\%), solo alcohólicos (18\%) y consumidores de drogas ilícitas (1\%). La mayoría $(60 \%)$ de ellos usaron drogas durante más de 10 años, más de tres veces por semana. Hubo una mayor prevalencia de lesiones con menor impacto en la salud (41\%), seguido de pacientes sin cambios estomatológicos (32\%) y lesiones con mayor impacto en la salud (27\%). Conclusión: A pesar de ser considerado una población en riesgo de desarrollar cáncer oral, el carcinoma de células escamosas (SCC) fue menos frecuente que la aparición de lesiones con potencial maligno, con predominio de cambios estomatológicos con menos impacto en la salud general de los pacientes.

Palabras clave: Dependientes químicos, Salud bucal, Medicina oral.

\section{INTRODUÇÃO}

Cada vez mais precocemente o uso de drogas se insere no cotidiano das populações. Os cinco levantamentos dos padrões de consumo de drogas pela população brasileira apontaram que o consumo de drogas é bastante alto entre crianças e adolescentes. Além disso, jovens e adultos tem aumentado cada vez mais o consumo de drogas, sendo fato de ocorrência frequente nas sociedades ocidentais (MINISTÉRIO DA SAÚDE, 2007).

Um levantamento epidemiológico realizado com 17 mil indivíduos na faixa etária entre 12 e 65 anos, por todo o território nacional, apontou que $9,9 \%$ dos entrevistados já fizeram, ao menos uma vez na vida o uso de alguma substância ilícita, sendo um percentual maior entre o sexo masculino, com $5 \%$ da população.

Estima-se que no Brasil, a maconha seja a droga ilícita mais consumida e 7,7\% da amostra estudada referiu já ter consumido. Este mesmo estudo revelou, ainda, que, mais da metade dessa população $(66,4 \%)$, já utilizou bebidas alcoólicas no mínimo uma vez, sendo a droga lícita mais comumente consumida (BASTOS FIPM, et al., 2017).

No Brasil, atualmente, entre as drogas mais frequentemente relatadas por usuários internados em processo de recuperação de dependência, estão o álcool, tabaco, maconha, crack e cocaína. A grande maioria realiza a associação de mais de um tipo de droga, não se limitando ao seu uso isolado (GUSMÃO PP, et al., 2017; TREVISAN ER e CASTRO SS, 2019). A toxicodependência promove alterações significantes nos tecidos orais, principalmente no que se refere às infecções bucais em decorrência da imunodeficiência em grande parte dos usuários. Um estudo sobre os efeitos deletérios de cada droga representa um desafio, uma vez que é muito frequente o uso combinado de substâncias (MATEOS-MORENO MV, et al., 2013).

Considerando que o álcool é a droga lícita mais consumida mundialmente, ressalta-se que abuso dessa substância promove efeitos deletérios à saúde bucal e sistêmica, devido à irritabilidade causada pelo contato com a mucosa oral, tendo sido também relacionada ao aumento de lesões cáries e doença periodontal, além dos seus efeitos carcinogênicos (COLODEL EV, et al., 2009; PÉRET ACA e BONATO KB, 2008).

Os indivíduos usuários de cocaína/crack apresentam quadros de xerostomia e/ ou redução do fluxo salivar. Ressalta-se, portanto, que a maior ocorrência de lesões orais nestes usuários pode ocorrer em detrimento das deficiências do sistema imune associadas ao contato da droga localmente e redução do fluxo salivar (COLODEL EV, et al., 2009). Há diversos efeitos sistêmicos e manifestações orais associadas ao uso de maconha, entre estas a uvulite, estomatite nicotínica, hiperplasia gengival semelhante aquela induzida por fenitoína, perda óssea na periodontite (NOGUEIRA-FILHO GR, et al., 2011). Além disso, usuários têm 
apresentado erosão dentária devido à Síndrome de Hiperêmese Canabinóide, condição em decorrência do uso dessa droga a longo prazo (COSSA F, et al., 2020). O câncer bucal e o uso do tabaco têm relação já bem estabelecida na literatura mundial. O consumo desta substância é considerado um fator de risco independente para o desenvolvimento do câncer, aumentando o risco relativo em sete a dez vezes quando comparado a indivíduos não fumantes (CONSOLARO RB, et al., 2010).

No geral, a ação local das drogas na cavidade oral apresenta diversos efeitos deletérios, tais como, gengivite, halitose, estomatite, bruxismo, desgastes dentais, além do aumento de lesões de cárie e distúrbios periodontais, podendo estar relacionados às deficiências nutricionais e negligência da higiene pessoal (GUPTA T, et al., 2012). De acordo com estudos epidemiológicos, houve um crescimento no uso indiscriminado de substâncias psicoativas. Dessa forma, o papel do cirurgião-dentista (CD) como profissional de saúde é atender às necessidades do paciente, sendo capacitado para tal. É preciso que o profissional se mantenha atualizado sobre as manifestações orais decorrentes do abuso de drogas, estando apto a diagnosticar e tratar esses pacientes de acordo com as necessidades apresentadas (MARQUES LARV, et al., 2016).

Considerando que o uso de substâncias químicas é um problema de saúde pública e, sabendo-se dos danos à saúde bucal ao qual essa população está exposta, verificou-se a necessidade de desenvolver um trabalho sobre o tema. Nesse sentido, este estudo tem por objetivo identificar a condição oral dos dependentes químicos atendidos em um Centro de Especialidades Odontológicas (CEO-Torre), localizado no município de João Pessoa- PB, traçar o perfil sociodemográfico, bem como determinar a prevalência de lesões/alterações estomatológicas nesses pacientes, de modo a abrir possibilidades para futuros estudos, tão escassos no atual contexto.

\section{MÉTODOS}

Por se tratar de uma pesquisa envolvendo seres humanos, foram atendidos os requisitos propostos pelo Conselho Nacional de Saúde/Ministério da Saúde, através da Resolução no 466/2012. Sendo assim, o projeto foi analisado pelo Comitê de Ética em Pesquisa em Seres Humanos do UNIPÊ sob no 49893115.1 .0000.5176 e aprovado pelo parecer de número 1.336.136. Caracteriza-se como um estudo observacional, retrospectivo e descritivo, apresentando características próprias de um estudo de campo de abordagem quantitativa.

O estudo foi desenvolvido no Centro de Especialidades Odontológicas (CEO-Torre) localizado na cidade de João Pessoa - PB, com uma amostra de 220 prontuários, dada por conveniência dentre os os pacientes assistidos no período de Dezembro de 2015 a Dezembro de 2016. Foram considerados como critérios para inclusão, prontuários de indivíduos atendidos no CEO-Torre, através do setor da Estomatologia, no intervalo de tempo descrito, que responderam positivamente, durante a anamnese, quanto ao uso de drogas. Foram excluídos da pesquisa os sujeitos cujos prontuários continham informações imprecisas e com falhas no seu preenchimento. De acordo com os dados coletados, estimou-se que foram atendidos neste período um número de 600 pacientes.

A coleta dos dados foi realizada através da transcrição das informações contidas nos prontuários, para o instrumento previamente elaborado pelos pesquisadores, que atendeu aos objetivos da pesquisa, buscando informações pertinentes ao estudo, tais como dados sociodemográficos, dados envolvendo as substâncias químicas utilizadas, histórico sistêmico e estomatológico relacionado ao uso de drogas. O material coletado foi analisado com base no enfoque do método quantitativo. Em sequência, os dados foram agrupados, apresentados em gráficos e/ou tabelas, e disponibilizados para a devida análise e discussão dos resultados à luz da literatura pertinente, através de estatística descritiva, por meio de números absolutos e percentuais.

\section{RESULTADOS}

Nesta pesquisa foram analisados 220 prontuários, dos quais 90 se enquadraram nos critérios de inclusão definidos para a seleção da amostra. Considerando o perfil sociodemográfico da população estudada, os pacientes usuários de drogas lícitas ou ilícitas apresentaram uma média de idade de 55,8 anos, sendo a 
mínima 17 e máxima 92. Houve predominância da faixa etária acima de 40 anos. Quanto ao gênero, houve prevalência do feminino. Observou-se maior percentual de pacientes que não apresentavam qualquer atividade financeira, e uma minoria de aposentados. Quanto à cor da pele, a maioria foi considerada parda (Tabela 1).

Tabela 1 - Distribuição dos pacientes segundo os dados sociodemográficos. João Pessoa, 2016.

\begin{tabular}{lll}
\hline Variável & N & $\%$ \\
\hline Sexo & 35 & 39 \\
\hline Masculino & 55 & 61 \\
Feminino & 90 & 100 \\
\hline Total & & \\
\hline Idade & 14 & 16 \\
\hline Menor ou igual a 40 anos & 76 & 84 \\
Maior que 40 anos & 90 & 100 \\
\hline Total & & \\
\hline Cor da pele & 22 & 24 \\
\hline Branco & 56 & 62 \\
Pardo & 8 & 9 \\
Negro & 4 & 4 \\
Não determinado & 90 & 100 \\
\hline Total & & \\
\hline Estado Civil & 35 & 39 \\
\hline Casado & 16 & 18 \\
Viúvo & 10 & 11 \\
Divorciado & 29 & 32 \\
Solteiro & 90 & 100 \\
\hline Total & & \\
\hline Ocupação & 34 & 38 \\
\hline Em atividade financeira & 43 & 48 \\
Sem atividade financeira & 13 & 14 \\
Aposentado & 90 & 100 \\
\hline Total & & \\
\hline Font Dados da pesquisa & & \\
\hline
\end{tabular}

Fonte: Dados da pesquisa, 2017.

As drogas mais utilizadas foram predominantemente o tabaco, seguindo-se o álcool associado ao tabaco, apenas álcool e o menor percentual de drogas ilícitas, as quais apresentam relação estreita com danos à saúde bucal, prevalecendo a maconha e o crack (Gráfico 1).

Gráfico 1 - Distribuição dos pacientes quanto ao uso de drogas lícitas ou ilícitas. João Pessoa, 2016.

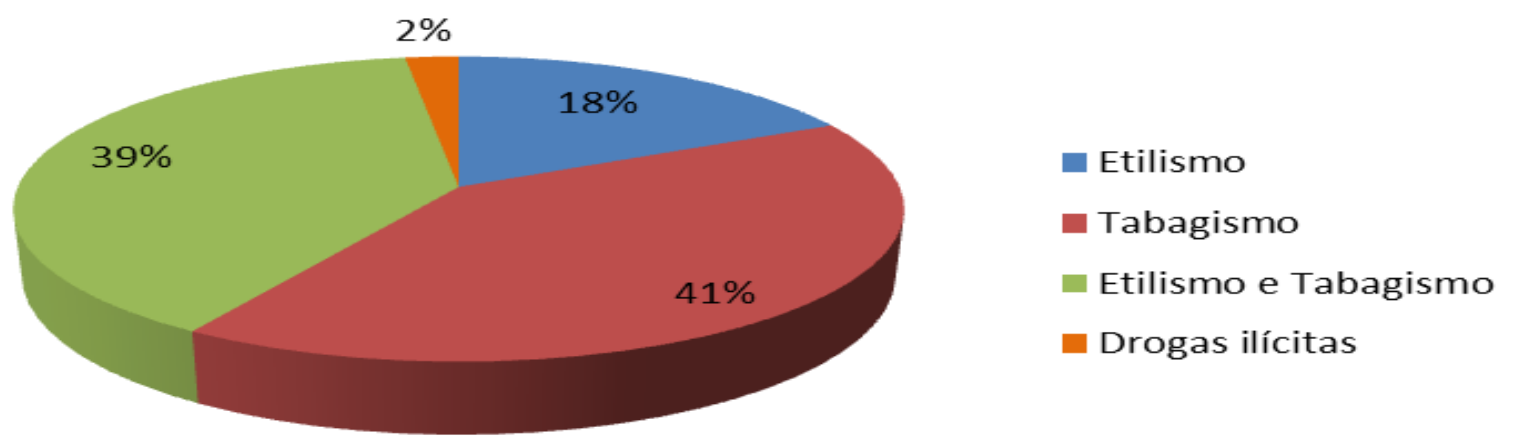

Fonte: Dados da pesquisa, 2017. 
Para a duração e frequência da dependência química foram considerados o tempo de uso e a periodicidade semanal, independente do tipo de droga consumida (Tabela 2). Nota-se que houve maior prevalência de pacientes que faziam uso de drogas por mais de 10 anos, e maior frequência correspondente a mais de três vezes por semana.

Tabela 2 - Distribuição dos pacientes quanto à duração e frequência de uso de drogas. João Pessoa, 2016.

\begin{tabular}{lcc}
\hline Variável & N & $\%$ \\
\hline Duração & & \\
\hline Mais de 10 anos & 54 & 60 \\
Menos de 10 anos & 25 & 28 \\
Não determinado & 11 & 12 \\
\hline Total & 90 & 100 \\
\hline Frequência & & \\
\hline Uma vez por semana & 8 & 9 \\
Duas a três vezes por semana & 25 & 28 \\
Mais de três vezes por semana & 57 & 63 \\
\hline Total & 90 & 100 \\
\hline Fon
\end{tabular}

Fonte: Dados da pesquisa, 2017.

No que diz respeito às alterações estomatológicas, neste estudo houve maior prevalência de pacientes com lesões sem maior impacto à saúde, seguindo-se aqueles sem alterações estomatológicas (Gráfico 2). A ocorrência de lesões impactantes incluiu as lesões com potencial de malignidade, como: adenoma pleomórfico, leucoplasia e líquen plano; o câncer de boca, representado pelo carcinoma de células escamosas (CCE); e doenças sistêmicas com repercussão bucal, o eritema multiforme e lúpus mucocutâneo crônico. Observou-se nessa amostra baixa prevalência de leucoplasia e CCE (Tabela 3).

Gráfico 2 - Distribuição dos pacientes quanto à prevalência das alterações estomatológicas. João Pessoa, 2016.

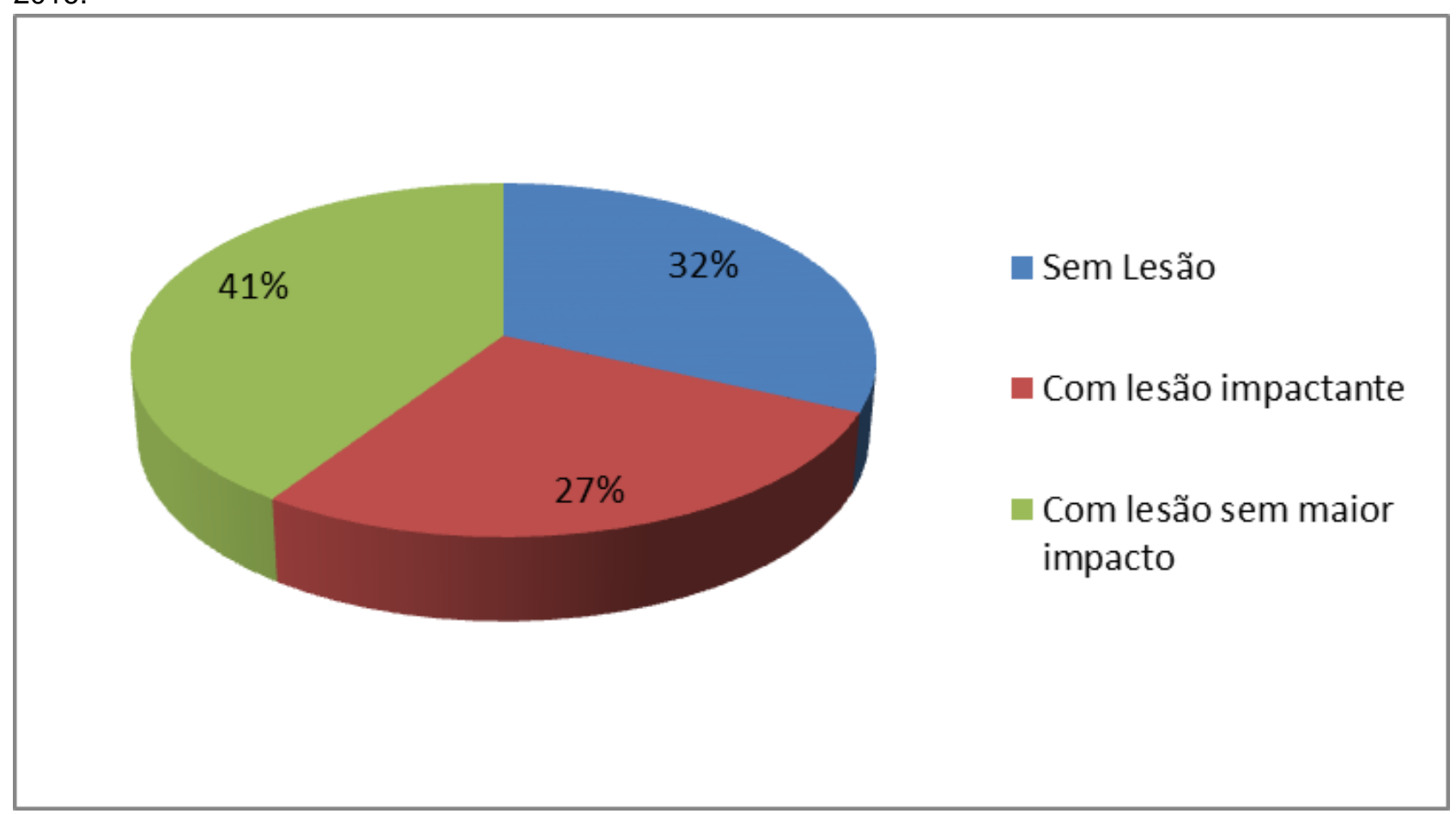

Fonte: Dados da pesquisa, 2017. 
Tabela 3 - Distribuição dos pacientes quanto às alterações estomatológicas. João Pessoa, 2016.

\begin{tabular}{|c|c|c|}
\hline Variável & $\mathbf{N}$ & $\%$ \\
\hline \multicolumn{3}{|l|}{ Alterações estomatológicas } \\
\hline Abscesso Periodontal & 1 & 1 \\
\hline Angina Bolhosa Hemorrágica & 1 & 1 \\
\hline Adenoma Pleomórfico & 3 & 3 \\
\hline Carcinoma de Células Escamosas & 7 & 8 \\
\hline Cisto Periapical & 1 & 1 \\
\hline Eritema Multiforme & 1 & 1 \\
\hline Estomatite Nicotínica & 1 & 1 \\
\hline Estomatite Protética & 3 & 3 \\
\hline Fenômeno de Extravasamento de Muco (não especificado) & 1 & 1 \\
\hline Fibroma Traumático & 2 & 2 \\
\hline Fibrolipoma & 1 & 1 \\
\hline Granuloma Piogênico & 1 & 1 \\
\hline Hiperceratose Friccional & 1 & 1 \\
\hline Hiperceratose Fibrosa & 1 & 3 \\
\hline Hiperplasia Fibrosa Inflamatória & 1 & 1 \\
\hline Hemangioma & 1 & 1 \\
\hline Infecção Herpética Recorrente & 1 & 1 \\
\hline Leucoplasia & 3 & 3 \\
\hline Linfadenopatia Reacional & 8 & 9 \\
\hline Líquen Plano & 9 & 10 \\
\hline Lipoma & 1 & 1 \\
\hline Lúpus Mucocutâneo Crônico & 2 & 2 \\
\hline Mucocele & 1 & 1 \\
\hline Pápula & 1 & 1 \\
\hline Papilite & 1 & 1 \\
\hline Papiloma & 1 & 1 \\
\hline Queilite Angular & 1 & 1 \\
\hline Rânula & 1 & 1 \\
\hline Reação Liquenóide & 1 & 1 \\
\hline Sialodenite associado a Mucocele & 1 & 1 \\
\hline Sem lesão & 31 & 35 \\
\hline Total & 90 & 100 \\
\hline
\end{tabular}

Fonte: Dados da pesquisa, 2017.

Quanto ao uso de medicamentos, observou-se que a maioria da amostra estudada não fazia uso de qualquer medicamento (83\%). Dentre os medicamentos utilizados destacaram-se os anti-hipertensivos (11\%), seguidos de ansiolíticos (4\%) e hipoglicemiantes (1\%).

\section{DISCUSSÃO}

A dependência das drogas é uma doença complexa, caracterizada por uma busca compulsiva, algumas vezes incontrolável, sendo que o seu uso persiste mesmo diante de consequências negativas. Estudos epidemiológicos sobre o consumo de drogas apontam que este fenômeno tem se constituído um problema dimensional na Saúde Pública (BARROS MA e PILLON SC, 2006).

Neste trabalho houve predominância da faixa etária acima de 40 anos. O estudo realizado por Pillon SC et al. (2010) observou um crescimento do uso de substâncias psicoativas por pessoas idosas, sendo considerado um problema de saúde pública. Para esses autores, embora a literatura aponte que a prevalência do uso de álcool tem sido maior entre os jovens e que diminui com a maturidade, o número de usuários de substâncias psicoativas continuará a aumentar com o crescimento da proporção da idade. 
Com relação ao gênero, houve prevalência do feminino, diferindo de uma pesquisa que visou abordar o perfil sociodemográfico e condição bucal de usuários de drogas, onde foi observada alta prevalência do gênero masculino, indicando um padrão de consumo de drogas ilícitas maior entre os homens (ALBINI MB, et al., 2015).

Por sua vez, estudo realizado sobre os fatores de risco para o câncer de boca, reforçou essa tendência ao afirmar que, devido a questões sociais, os homens estão mais envolvidos com os fatores de risco para o câncer bucal, como o álcool e o fumo, do que as mulheres (GORSKY M, et al., 2004). Nesta pesquisa, a predominância de mulheres pode sugerir uma maior procura, por parte deste grupo, aos serviços de Odontologia, refletindo um maior cuidado com a saúde bucal em relação aos homens.

As drogas mais utilizadas neste estudo foram, predominantemente, o tabaco, seguindo-se o álcool associado ao tabaco, apenas álcool e drogas ilícitas, as quais apresentam relação estreita com danos à saúde bucal, visto que a cavidade oral é a porta de entrada principal desses agentes, tornando-se bastante susceptível ao sinergismo e aos efeitos nocivos do uso dessas substâncias (SORDI MB, et al., 2017). Sabese que a toxicodependência causa alterações significativas no ambiente bucal (MARQUES LARV, et al., 2016). O uso do tabaco de mascar "in natura" ou uma mistura traz significativos riscos para a saúde. Além disso, os fumantes de cigarro de palha apresentam níveis maiores de dependência da nicotina e risco aumentando de desenvolver doenças relacionadas ao tabaco (MINISTÉRIO DA SAÚDE, 2006).

Miotto MHMB et al. (2017) realizaram um estudo transversal que objetivou determinar o impacto produzido por problemas orais na qualidade de vida em dependentes químicos, considerando que o álcool e o tabaco trazem maiores e mais graves consequências para a saúde pública, sendo as drogas mais consumidas no mundo. Diante disso, é preciso a incorporação efetiva dos profissionais da odontologia em projetos de recuperação e promoção da saúde oral, com a finalidade de melhorar a qualidade de vida.

As alterações bucais em usuários de droga são frequentes. $O$ uso prolongado dessas substâncias e as alterações sistêmicas são alguns dos fatores responsáveis por tal condição. Além disso, os fatores sociais também contribuem para o desenvolvimento e evolução de tais patologias, tais como: marginalizalação, baixa auto-estima, má-higiene bucal, dificuldade de acesso aos serviços odontológicos. Ademais, hábitos alimentares insatisfatórios e a busca por alimentos açucarados após o uso de drogas também contribui para a má qualidade de higiene e consequentemente da saúde bucal (ARORA PC, et al., 2019).

O consumo de substâncias químicas influencia diretamente na qualidade de vida de indivíduos dependentes, pois pode acarretar prejuízos físicos, psicológicos, sociais e legais (SILVEIRA C, et al., 2013; MACIEL SC, et al., 2018), além de interferir negativamente na saúde bucal e geral do indivíduo (MIOTTO MHMB, et al., 2017). Para Silveira $C$ et al. (2013), uma possível dificuldade enfrentada pelos dependentes químicos é a sua própria aceitação na sociedade e, por alimentarem esse sentimento, o vício também é alimentado, evoluindo com um quadro de saúde agravado.

Mediante o exposto, quanto mais precoce o tratamento for iniciado mais chances de ressocialização o indivíduo apresenta, além de diminuir os riscos à saúde. No entanto, isso acarretará também danos no contexto familiar, uma vez que gera sofrimento àqueles que vivem de forma indireta a dependência química. Atrelado a isso, verifica-se o consequente aumento na sobrecarga de trabalho dos familiares para suprir as necessidades que a situação impõe, influenciando negativamente na qualidade de vida no âmbito familiar (MACIEL, SC et al., 2018).

O presente estudo mostrou que o uso de álcool associado ao tabaco correspondeu a $39 \%$ da amostra. Um fato a ser considerado na etiologia das neoplasias da cavidade oral é a magnitude do risco resultante da interação de drogas, cujos efeitos adversos não são simplesmente aditivos, mas sim multiplicativos, atingindo níveis elevados naqueles indivíduos com alto consumo simultâneo dessas substâncias (SZYMANSKA K, et al., 2011).

O tabaco é um carcinógeno potente e, juntamente com o uso crônico do álcool, torna-se o mais importante fator de risco para o desenvolvimento do câncer de boca (NEVILLE BW, et al., 2009). Nesta pesquisa o etilismo isolado correspondeu a $18 \%$ da amostra. O etilismo resulta em graves consequências, sendo um dos agentes químicos relacionados ao desenvolvimento de neoplasias malignas (ALVES DM, et al., 2013). 
Determinadas concentrações de álcool causam aumento da permeabilidade da mucosa bucal, potencializando a penetração de carcinógenos. Além disso, essa substância é responsável por um aumento na proliferação epitelial, bem como pela modificação do seu processo de maturação. Outras alterações, como redução da capacidade de reparo de DNA, distúrbios do sistema imune e do estado nutricional podem contribuir na sua relação com o desenvolvimento do câncer bucal (CARRARD VC, et al., 2008).

Existem controvérsias se o álcool, por si só, exerce efeito carcinogênico. Evidências apontam que o álcool tem efeito aditivo, ou seja, seu efeito carcinogênico é considerado quando associado ao fumo (TOMMASI MH, 2013). No entanto, o Ministério da Saúde (2020) reconhece o efeito cancerígeno do etanol e seu principal metabólito, o acetaldeído, sobre as células, embora o mecanismo não seja, ainda, bem definido.

Neste trabalho houve uma menor prevalência de usuários de drogas ilícitas, dentre estas, prevaleceram à maconha e o crack, contrapondo-se outras pesquisas, onde houve uma elevada frequência do consumo de crack, suplantando o consumo de maconha e cocaína, ganhando destaque em função dos seus efeitos devastadores sobre os usuários e o seu ambiente social (SANCHEZ ZM, et al., 2005). A razão da baixa prevalência nesse estudo pode estar relacionada ao fato de que nem sempre o usuário revela a real condição, quer por constrangimento quer pelo receio de sofrer discriminação.

Nos últimos cinquenta anos acumularam-se inúmeras evidências de que o consumo de bebidas alcoólicas, tanto no que diz respeito à quantidade quanto à duração, aumenta o risco do desenvolvimento de tumores de cabeça e pescoço (cavidade oral, faringe e laringe) e esôfago (BANN R, et al., 2007). Tais achados são corroborados por Waszkiewicz $\mathrm{N}$ et al. (2012), os quais afirmam que o etilismo frequente resulta em graves consequências à saúde, sendo considerado um dos agentes químicos relacionados ao desenvolvimento de neoplasias malignas bucais, incluindo o câncer de boca. Com relação ao tabaco, segundo Lopes CBF et al. (2012), indivíduos que fazem uso prolongado e constante de cigarro têm mais chances de desenvolver câncer bucal.

Os autores supracitados acrescentam, ainda, que, em relação à população geral, os indivíduos que se encontram em estado de drogadição exibem uma acentuada prevalência de lesões bucais com potencial de malignização, tais como: queilite actínica, leucoplasia, eritroplasia e líquen plano (LOPES CFB, et al., 2012). No Brasil, o CCE bucal é mais associado ao tabaco, sob a forma de cigarro, e à bebida alcoólica. O CCE da cavidade oral e orofaringe representam $2 \%$ de todos os casos de câncer registrados no mundo, e em $50 \%$ desses casos os pacientes vão a óbito devido a complicações da doença (FELLER L e LEMMER J, 2012).

No presente estudo verificou-se uma maior prevalência de pacientes que faziam uso de drogas por mais de 10 anos em uma frequência correspondendo a mais de três vezes por semana. Apesar de ter sido observado um maior percentual de tabagistas, seguido da associação do tabagismo e etilismo, houve baixa prevalência de lesões potencialmente malignas e do câncer de boca, este último representado por um percentual equivalente a $8 \%$.

Tal resultado vem reforçar a multifatoriedade desta neoplasia, evidenciando que, apesar do álcool e tabaco serem os principais fatores de risco para o desenvolvimento do câncer de boca (assim como é reconhecida a associação da leucoplasia com o uso do tabaco), existem outros fatores implicados na sua etiologia, incluindo as deficiências nutricionais, agentes biológicos (vírus) e fatores genéticos.

Quanto ao uso de medicamentos, observou-se neste estudo que a maioria da amostra não fazia uso de qualquer medicamento. É difícil relacionar determinada alteração bucal encontrada nos pacientes com uma droga específica, pois, alguns dos usuários fazem uso concomitante de mais de um tipo de droga, ou estão em tratamento com medicamentos suscetíveis de causar alterações bucais. Além disso, o uso de vários tipos de drogas simultaneamente poderia potencializar o aparecimento de lesões bucais (SANTOS GL, et al., 2010).

As substâncias mais utilizadas como calmantes são os benzodiazepínicos, como por exemplo, o Diazepam. Nesse sentido, observou-se, na amostra estudada, uma baixa prevalência do uso de ansiolíticos. Ainda de acordo com os autores supracitados, os dependentes crônicos em sedativos e antidepressivos podem apresentar hipossalivação, com desenvolvimento de xerostomia (O'SULLIVAN EM, 2012). 
Cada substância tem seu poder devastador sobre os tecidos bucais. Logo, os usuários de drogas, lícitas ou ilícitas, podem ser considerados como um grupo de risco para o desenvolvimento de alterações bucais, incluindo-se lesões com potencial de malignidade e o câncer de boca (COLODEL EV, et al., 2009).

O diagnóstico precoce de câncer na cavidade oral é um fator determinante para garantia de um prognóstico favorável, tendo em vista que quanto antes o tratamento for iniciado mais alto serão as chances de cura. Diante disso, é importante que os serviços de saúde da atenção básica estejam preparados e capacitados para conduzir o caso e oferecer atenção integral ao paciente suspeito e/ou diagnosticado, utilizando de estratégias e medidas de prevenção e promoção, a fim de reduzir a mortalidade e o número de novos casos (RAIMUNDO et al., 2019; RODRIGUES et al., 2019).

Rodrigues LV et al. (2019) realizaram um estudo a fim de descrever as ações em saúde bucal frente ao câncer de boca pelas equipes de saúde da atenção básica da região do Nordeste, e de acordo com os resultados, concluiu que as equipes avaliadas necessitam de melhores estratégias de detecção, encaminhamento e condução dos casos. Em outro estudo, Leôncio LL et al. (2015) avaliou a conduta dos cirurgiões-dentistas diante do diagnóstico de pacientes com doenças da cavidade oral, atendidos no sistema público de saúde do município de Patos, Paraíba, afirmando que, os profissionais não seguem um protocolo específico para encaminhamento ou tratamento desses pacientes. Para Santos IV et al. (2011) o cirurgiãodentista é fundamental na prevenção de lesões malignas da cavidade bucal, em essencial quando se procede em níveis de prevenção primária e secundária.

Recomenda-se a realização de estudos longitudinais do tema em questão, a fim de edificar as reais condições de saúde bucal dos dependentes químicos e o real impacto dessas substâncias na cavidade oral. Os cirurgiões-dentistas precisam estar aptos ao atendimento desses pacientes considerando os sinais clínicos que possam surgir em decorrência do uso de substâncias químicas, para então, realizar o condicionamento terapêutico adequado. Nesse contexto, visando à reabilitação desses pacientes, planos de ações de prevenção a agravos e promoção a saúde são essenciais.

\section{CONCLUSÃO}

Diante dos resultados apresentados, pode-se concluir que a amostra se caracterizou por pacientes de meia idade, predominando o gênero feminino, pardos, casados e a maioria sem qualquer atividade financeira. Observou-se ainda, um percentual considerável de pacientes que faziam uso de tabaco e da associação álcool e tabaco, fatores de risco importantes para o desenvolvimento do câncer de boca. Contudo, o CCE foi pouco prevalente, ainda menos que a ocorrência de lesões com potencial de malignidade, com maior frequência de alterações estomatológicas, sem grande impacto à saúde geral dos pacientes. Esse estudo torna-se relevante por trazer subsídios para o planejamento de políticas públicas, principalmente desse município da Paraíba, que visam promover ações preventivas relacionadas à saúde bucal.

\section{REFERÊNCIAS}

1. ALBINI MB, et al. Perfil sociodemográfico e condição bucal de usuários de drogas em dois municípios do Estado do Paraná, Brasil. Rev. Odontol. UNESP, 2015; 44(4): 244-249.

2. ALVES DM, et al. Avaliação da Ação do Uso de Drogas na Saúde Bucal de Dependentes Químicos. Colloquium Vitae, 2013; 5(1): 40- 58.

3. ARORA PC, et al. Oral Health Behavior and Treatment Needs among Drug Addicts and Controls in Amritsar District: A Case-controlled Study. J Neurosci Rural Pract, 2019; 10(2): 201-206.

4. BANN R, et al. Carcinogenicity of Alcoholic beverages. Lancet Oncology, 2007; 8(4): 292-293.

5. BARROS MA, PILLON SC. Programa Saúde da Família: desafios e potencialidades frente ao uso de drogas. Revista Eletrônica de Enfermagem, 2006; 8(1).

6. BASTOS FIPM, et al. III Levantamento Nacional sobre o uso de drogas pela população brasileira. Ministério da Saúde, Fundação Oswaldo Cruz, 2017.

7. BRASIL. Ministério da Saúde. A política do Ministério da Saúde para a atenção integral a usuários de álcool e outras drogas. $2^{\mathrm{a}}$ ed revisada e ampliada. Brasília: Ministério da Saúde, 2007. Série B. Textos básicos da Saúde.

8. BRASIL. Ministério da Saúde. Instituto Nacional de Câncer José Alencar Gomes da Silva (INCA). Estimativa 2020: incidência de câncer no Brasil. Rio de Janeiro. 2020. 
9. BRASIL. Ministério da Saúde. Instituto Nacional de Câncer. 2006.

10. CARRARD VC, et al. Alcohol and Oral Cancer: Comments on Related Mechanisms. Rev Bras Cancerologia, 2008; 54(1): 49-56.

11. COLODEL EV, et al. Alterações bucais presentes em dependentes químicos. Rev. Sul- Brasileira de odontologia, 2009; 6(1): 44-48.

12. CONSOLARO RB, et al. O tabaco é um dos principais fatores etiológicos do câncer bucal: conceitos atuais. Rev. Odontológica de Araçatuba, 2010; 31(2): 63-67.

13. COSSA F, et al. Oral manifestations in drug users: A review. J. Clin Exp Dent, 2020; 12(2): e193-e200.

14. FELLER L, LEMMER J. Oral Leukoplakia as It Relates to HPV Infection: A Review. Int J Dent, 2012.

15. GORSKY M, et al. Carcinoma of the Tongue: a Case Series Analysis of Clinical Presentation, Risk Factors, Staging, and Outcome. Oral Surg. Oral Med. Oral pathol. Oral Radiol endond, 2004; 98(5): 546-52.

16. GUPTA T, et al. Oral health status of a illicit drug users in Delhi, India. Community Dent Health, 2012; $29(1)$ : $49-54$.

17. GUSMÃO PP, et al. Perfil epidemiológico de uma população de usuários de drogas de Anápolis, Goiás. Revista Educação em Saúde, 2017; 5(1): 28-37.

18. LEÔNCIO LL, et al. Diagnóstico e encaminhamento de pacientes com doenças bucais no serviço público de saúde de Patos-PB: atuação do cirurgião-dentista na referência e contra referência. Arq Odontol, Belo Horizonte, 2015; 51(4): 210-215.

19. LOPES CFB, et al. Concomitant consumption of marijuana, alcohol and tabacco in oral squamous cell carcinoma development and progression: recente advances and challenges. Arch Oral Biol, 2012; 57(8): 1026-133.

20. MACIEL SC, et al. Cuidadoras de Dependentes Químicos: um estudo sobre a sobrecarga familiar. Psicologia: Teoria e Pesquisa, 2018; 34: 1-10.

21. MARQUES LARV, et al. Abuso de drogas e suas consequências na saúde bucal: uma revisão de literatura. FOL: Faculdade de Odontologia de Lins/ Unimep, 2016; 26(1): 29-35.

22. MATEOS-MORENO MV, et al. Dental profile of a community of recovering drug addicts: Biomedical aspects. Retrospective cohort study. Med Oral Patol Oral Cir Bucal, 2013; 18(4): e671-e679.

23. MIOTTO MHMB, et al . Impacto dos Problemas Orais na Qualidade de Vida de Dependentes Químicos em Recuperação num Centro de Tratamento. Port J Public Health, Lisboa, 2017; 35(1): 30-36.

24. NEVILLE BW, et al. Oral and maxillofacial pathology. 3.ed. Philadelphia: Saunders Elsevier; 2009.

25. NOGUEIRA-FILHO GR, et al. Impacto of cannabis sativa (marijuana) smoke onde alveolar bone loss: a histometric study in: rats. J Periodontol, 2011; 82(11): 1602- 1607.

26. O'SULLIVAN EM. Dental Health of Irish alcohol/drug abuse treatment centre residentes. Community Dent Health, 2012; 29(4): 263-267.

27. PÉRET ACA, BONATO KB. A participação do dentista na equipe multidisciplinar para o tratamento do paciente alcoolista. Arq. Bras. Odontol, 2008; 4(2): 70-5.

28. PILLON SC, et al. Perfil dos idosos atendidos em um centro de atenção psicossocial: álcool e outras drogas. Esc. Anna Nery, Rio de Janeiro, 2010; 14(4): 742-748.

29. RAIMUNDO DD, et al. manifestações clínicas e sociodemográficas em trabalhadores com câncer bucal. Rev Enferm Ufpe On Line, Recife, 2019; 13(5):1412-1419.

30. RODRIGUES LV, et al. Oral health actions in the primary health care network of northeastern brazil in relation to oral cancer. RGO, Rev. Gaúch. Odontol, 2019; 67:1-9.

31. SANCHEZ ZM, et al. Razões para o não uso de drogas ilícitas entre jovens em situação de risco. UNIFESP. Revista Saúde Pública, 2005; 39(4): 599-605.

32. SANTOS GL, et al. Fumo e álcool como fatores de risco para o câncer bucal. Odontol. Clín.-Cient. Recife, 2010; 9(2): 131-133.

33. SANTOS VI, et al. O papel do cirurgião-dentista em relação ao câncer de boca. Odontol. Clín.-Cient, 2011; 10(3): 207-210.

34. SILVEIRA C, et al. Qualidade de vida, autoestima e autoimagem dos dependentes químicos. Ciência \& Saúde Coletiva, 2013; 18(7): 2001-2006.

35. SORDI MB, et al. Oral health assessment for users of marijuana and cocaine/crack substances. Braz. Oral Res, 2017; 31(102): 1-12.

36. SZYMANSKA K, et al. Alcohol and tobacco, and the Risk of Cancers of the Upper Aerodigestive tract in Latin America: a Case-control Study. Cancer Causes Control, 2011; 22(7): 1037-46.

37. TOMMASI MH. Diagnostico em patologia bucal. 4.ed. Rio de Janeiro: Elsevier, 2013.

38. TREVISAN ER, CASTRO SS. Centros de Atenção Psicossocial - álcool e drogas: perfil dos usuários. Saúde debate, 2019; 43(121): 450-463.

39. WASZKIEWICZ N, et al. The effect of chronic alcohol intoxication and smoking on the activity of oral peroxidase. Folia Histochem Cytobiol, 2012; 50(3): 450-455. 